\title{
Special Section on DRS 25th Anniversary
}

\author{
Reza Shaker ${ }^{1}$
}

Published online: 25 January 2017

(c) Springer Science+Business Media New York 2017

Dysphagia research has come a long way since the first observation of the deglutitive dynamics and kinematics involved in transferring a bolus from the mouth to the stomach using the fascinating but rudimentary early radiographic techniques. Care of dysphagic patients has also come a long way from the days with no option but malnutrition, chest infection, and starvation. Research and discovery have come to the rescue, although not to the complete defeat of the menace but to a great extent have improved quality of life, extended life, improved nutrition, and prevented infection. The reciprocal duet of taking a clinical question in the form of hypothesis to the research laboratory and bringing a new discovery to be tested in clinic has taken hold. So has the recognition that dysphagia and its related research is inherently multidisciplinary and in clear need of the infusion of knowledge and expertise from multiple disciplines to advance further, hence the birth of the nascent discipline of deglutology.

The Dysphagia Research Society (DRS) undoubtedly has been an effective leader in promoting the multidisciplinary culture in dysphagia research and care. Indeed, the multidisciplinary structure of DRS has been instrumental in its overall success and in promoting its goals. A great deal of credit for this success goes to the visionary leaders from multiple disciplines who were consulted about forming the DRS in 1990-1991 and responded positively to my invitations and joined me in the Board of Directors working collaboratively to formalize the society in November of 1992 during its inaugural meeting in Milwaukee, Wisconsin.

\footnotetext{
Reza Shaker

rshaker@mcw.edu

1 Milwaukee, WI, USA
}

Similarly, a great deal of credit goes to the strong leaders who subsequently served in different capacities and/or lead and nurtured the society from infancy to its current state of maturity. Unfortunately two of the members of the Board of Directors, Drs. Jerry Dodds and Martin Donner did not live to see the inauguration for the society but others like Drs. Bronwyn Jones, Clarence Sasaki, Jeri Logeman, Walter Hogan, Donald Castel, Konrad Schulze, Barbara Sonnies, and Detlaf Beiger saw the formation of the society through and a number of them served as its future presidents.

The Dysphagia Research Society in the 25 years of its existence along with its younger sister societies in Europe, Japan, South America, and Southeast Asia has galvanized the community of researchers and clinicians and provided them with appropriate forum for exchange of ideas, sharing, and critiquing of their findings and development of improved approach to dysphagia care. Proudly the journal, Dysphagia, has uniquely partnered in all these effort for all these years, provided an international medium for dissemination of research finding with firm dedication to advancing the art and science of deglutology.

Dysphagia research has come a long way but alas, still lags behind other disciplines. The level of research efforts and research funding does not seem to match the enormity of unmet needs for novel therapeutics for dysphagic patients. Innovative research in dysphagia rehabilitation is scarce; regenerative medicine has not yet seriously found its way into dysphagia research, and despite rapid growth of aging population and frailty, research on developing swallowing health maintenance programs is lacking. What is also lacking, is a concerted effort to engage the community of stakeholders, patients, families, advocacy groups, and relevant industry and government agencies along with the investigators in addressing the research needs for better dysphagia care and implementing a team science model for dysphagia research. 
In order to remedy these shortcomings, the DRS leadership in this issue has chartered new territories and has identified areas in need of investigation. This important document addresses the entire deglutitive function from the cerebral cortex to the myenteric plexus, it considers all phases of swallowing from oral phase and mastication to the esophageal phase and trans-LES flow. It provides an important guidance and indicates strong commitment. To be successful, however, in advancing the field, commitment is also needed to engage the federal funding agencies and philanthropic organizations, in an effort to demonstrate the impact of dysphagia on the well-being of our citizens and the importance of basic, clinical, and translational research in swallowing and swallowing disorders for the overall health of the nation.

On the occasion of the 25th anniversary of formation of DRS, Dysphagia has dedicated the February 2017 issue to the celebration of this silver anniversary and our long standing partnership. A number of distinguished scholars have joined this dedication by contributing perspectives on various topics related to dysphagia research and care. We are deeply grateful for their efforts and support.

On a personal note, Happy 25th Birthday DRS. It gives me great pleasure to see you all mature, all vibrant and strong with a great purpose, a clear direction, promoting advancing health through research and discovery, galvanizing and energizing the next generation of investigators to carry the mantel, and remaining the advocate and the voice of millions upon millions of dysphagic patients worldwide. May your service never cease.

Reza Shaker MD 